\title{
Expression levels of vascular endothelial cell growth factor and microRNA-210 are increased in medulloblastoma and metastatic medulloblastoma
}

\author{
YONG GAO, PUXIAN LI, ZHENRUI LIU, XINGTAO DIAO and CHUNYUN SONG \\ Department of Neurosurgery, People's Hospital of Laiwu, Laiwu, Shandong 271100, P.R. China
}

Received January 14, 2015; Accepted September 1, 2015

DOI: $10.3892 /$ etm.2015.2810

\begin{abstract}
The present study aimed to investigate the roles of the vascular endothelial cell growth factor (VEGF) and micro (mi)RNA-210 in the metastasis of primary medulloblastoma (MB) tumors. A total of 86 adult patients diagnosed with cerebellar MB were enrolled in the present study, of which 11 patients had metastatic MB in the subarachnoid space. The following samples were collected: MB primary tumor tissue, MB secondary tumor tissue, tumor adjacent tissues and cerebrospinal fluid (CSF). Immunohistochemical analyses of the tissue samples were conducted in order to detect patterns of VEGF expression. In addition, the expression levels of VEGF mRNA and miRNA-210 were analyzed using reverse transcription-quantititative polymerase chain reaction, and western blot analyses were used to investigate VEGF protein expression levels. The positive expression rate of VEGF was significantly higher in $\mathrm{MB}$ tumor tissue, as compared with adjacent tissues $(\mathrm{P}<0.01)$. In addition, VEGF mRNA and protein expression levels in MB primary and secondary tumor tissues, and in the CSF of patients with metastatic MB, were significantly upregulated, as compared with tumor adjacent tissues and the CSF of patients with non-metastatic MB, respectively $(\mathrm{P}<0.01)$. miRNA-210 expression levels were significantly upregulated in MB tumor tissues, the CSF of patients with metastatic $\mathrm{MB}$ and in tumor tissues of metastatic $\mathrm{MB}(\mathrm{P}<0.01)$. In the present study, the expression levels of VEGF and miRNA-210 were upregulated in patients with MB and metastatic MB; thus suggesting that miRNA-210 may promote the metastasis of MB primary tumors by regulating the expression of VEGF.
\end{abstract}

Correspondence to: Dr Puxian Li, Department of Neurosurgery, People's Hospital of Laiwu, 001 Xuehu Avenue, Laiwu, Shandong 271100, P.R. China.

E-mail: 1px222@126.com

Key words: miRNA-210, medulloblastoma, vascular endothelial cell growth factor

\section{Introduction}

Medulloblastoma (MB) is a malignant epithelial tumor of the central nervous system, which often occurs in children (1). The underlying factors contributing to $\mathrm{MB}$ are currently unknown. MB primary tumors are able to develop in any part of the brain; however, they predominantly occur in the cerebellar vermis, above the top of the fourth ventricle. The initial clinical symptoms of MB include headaches, vomiting and unstable walking, which may progress to diplopia, ataxia and vision loss due to increased intracranial pressure and cerebellar damage (2).

Tumorigenesis, development, invasion, metastasis, and malignancy are closely associated with angiogenesis (3). The vascular endothelial cell growth factor (VEGF) is an effective pro-angiogenic growth factor and is an important regulator of angiogenesis, in which new blood vessels are formed from existing ones in order to increase blood supply (4). Angiogenesis has a key role in the malignant transformation of normal tissues (5), and alterations in the expression levels of numerous vascular growth factors, including VEGF, have been detected during the development of various tumor types (6). Furthermore, VEGF has been associated with the development of numerous diseases, including coronary heart disease (7), cardiac X syndrome (8), hypertension (9), cerebrovascular disease (10), and diabetic nephropathy (11).

Previous studies have detected an association between upregulation of micro (mi)RNA-210 expression levels and elevated expression levels of VEGF in kidney tissue samples, during bone necrosis, and in glioblastoma (12-14). However, to the best of our knowledge, the relationship between miRNA-210 and VEGF in patients with MB has yet to be investigated. In the present study, the expression levels of miRNA-210 and VEGF in $\mathrm{MB}$ primary and secondary tumor tissues, tumor adjacent tissues and in the cerebrospinal fluid (CSF) were detected, in order to investigate the association between miRNA-210 and VEGF, and their roles in MB metastasis.

\section{Materials and methods}

Subjects. A total of 86 adult patients with cerebellar MB, who were admitted to the People's Hospital of Laiwu (Laiwu, China) between January 2011 and June 2014, were enrolled in the present study. Of the 86 patients, 50 were male and 36 were 
female. The age range was $18-46$ years, with a mean age of $35.6 \pm 8.6$ years. All patients underwent surgery to remove the tumor. Following the initial surgery, MB metastasis to the subarachnoid space occurred in 11 patients, including 5 male and 6 female patients. The age range of these 11 patients was 21-39 years, with a mean age of 29.3 \pm 8.1 years. The metastatic MB tissue was similarly removed by surgery. The tumor tissues of primary $\mathrm{MB}$ and metastatic $\mathrm{MB}$, tumor adjacent tissues and CSF were collected. Written informed consent was obtained from all patients prior to the study. The study was approved by the Ethics Review Board of the People's Hospital of Laiwu.

Reagents and instruments. The miRcute miRNA Isolation kit, miRcute miRNA cDNA First Strand Synthesis kit, miRcute miRNA Quantitative Fluorescence Detection kit, SuperReal PreMix (SYBR Green), and TIANScript II cDNA First Strand Synthesis kit, were all obtained from Tiangen Biotech Co., Ltd. (Beijing, China). Rabbit anti-human polyclonal VEGF antibody (cat. no. ab46154), rabbit anti-human polyclonal $\beta$-actin antibody (cat. no. ab129348), horseradish peroxidase (HRP)-conjugated sheep anti-rabbit immunoglobulin (Ig)G (cat. no. ab6721), and goat anti-rabbit biotinylated secondary monoclonal antibody (cat. no. ab128978) were purchased from Abcam (Cambridge, MA, USA). TRIzol ${ }^{\circledR}$ reagent was obtained from Liaoning Yisheng Biological Pharmaceutical Co., Ltd. (Shenyang, China). The bicinchoninic acid (BCA) protein assay kit was purchased from Zhong Ke Rui Tai Biotech Co. (Beijing, China). The Image Lab 3.0 software and high-performance iQ5 Real-Time PCR Detection system were obtained from Bio-Rad Laboratories, Inc. (Hercules, CA, USA). Primers were designed using Primer Premier 5.0 software (Premier Biosoft International, Palo Alto, CA, USA) and were synthesized by Sangon Biotech Co., Ltd. (Shanghai, China).

Immunohistochemistry. MB tumor tissues and tumor adjacent tissues were fixed with $10 \%$ formalin and embedded in paraffin. Tissues were cut into $4 \mu \mathrm{m}$ sections, which were then dewaxed in graded xylene and dehydrated in graded alcohols. In order to inactivate endogenous peroxidase, tissue sections were incubated with $3 \%$ hydrogen peroxide for $10 \mathrm{~min}$ at room temperature. Antigen retrieval was performed in a microwave $\left(92^{\circ} \mathrm{C}\right.$ for $15 \mathrm{~min}$ ). After blocking in $5 \%$ goat serum (Beijing Zhongshan Golden Bridge Biological Technology Co., Ltd., Beijing, China), rabbit anti-human VEGF polyclonal antibody (dilution 1:200) was added and incubated at room temperature for $1 \mathrm{~h}$, prior to incubation with biotinylated goat anti-rabbit antibody (dilution $1: 1,000$ ) at $37^{\circ} \mathrm{C}$ for $30 \mathrm{~min}$. The tissue sections were then incubated with 3,3-diaminobenzidine chromogenic substrate reagent (Abcam) and counterstained with haematoxylin (Abcam). Following differentiation with hydrochloric acid and the dimethylbenzene transparency procedure, tissue sections were mounted with neutral gum.

Evaluation of immunohistochemical staining results. Tissue sections were observed under a microscope (magnification, 400x; Olympus BX50; Olympus Corporation, Tokyo, Japan). Cells with brown or tan granules in the cytoplasm or on the membrane were defined as positive. Images of five fields at high-magnification were randomly captured and positive cells were counted. At least 100 cells were counted. The positive rate corresponded to the ratio of the number of positive cells to the total number of cells counted.

Reverse transcription-quantitative polymerase chain reaction $(R T-q P C R)$. The expression levels of VEGF mRNA and miRNA-210 were detected in tissue and CSF samples using RT-qPCR. Prior to RNA extraction, tissue samples were homogenized using a homogenizer (PRO 200 homogenizer; Pro Scientific, Inc., Oxford, CT, USA). For analysis of VEGF mRNA expression levels, total RNA was extracted from tumor tissues and tumor adjacent tissues using TRIzol ${ }^{\circledR}$ reagent, and the total RNA was reverse transcribed into cDNA. The primers used were as follows: Forward, 5'-TTGCCTTGCTGC TCTACCTC-3' and reverse, 5'-AAATGCTTTCTCCGCTCT GA-3' for VEGF; and forward, 5'-TGACGTGGACATCCG CAAAG-3' and reverse, 5'-CTGGAAGGTGGACAGCGA GG-3' for $\beta$-actin. $\beta$-actin was used as an internal control. PCR-iQ5 thermal cycler (Bio-Rad Laboratories, Inc.) was used to perform PCR. The PCR cycling procedures were as follows: Pre-denaturation at $94^{\circ} \mathrm{C}$ for $2 \mathrm{~min}, 35$ cycles of denaturation at $94^{\circ} \mathrm{C}$ for $30 \mathrm{sec}$, annealing at $55^{\circ} \mathrm{C}$ for $30 \mathrm{sec}$ and extension at $71^{\circ} \mathrm{C}$ for $1 \mathrm{~min}$, and a final extension at $71^{\circ} \mathrm{C}$ for $2 \mathrm{~min}$. The $2^{-\Delta \Delta \mathrm{Ct}}$ method (15) was used to calculate the relative expression levels of VEGF and $\beta$-actin.

The following primers were used to analyze the expression levels of miRNA-210 in the CSF: Forward, 5'-CTGTGCGTGTGACAGCGGCTGA-3' and reverse, 5'-GCGAGCACAGAATTAATACGAC-3' for miRNA-210; and forward, 5'-CGCTTCGGCAGCACATATACTA-3' and reverse, 5'-CGCTTCACGAATTTGCGTGTCA-3' for U6. U6 was used as an internal control. The PCR cycling procedures were as follows: Pre-denaturation at $95^{\circ} \mathrm{C}$ for $10 \mathrm{~min}, 40$ cycles of denaturation at $95^{\circ} \mathrm{C}$ for $15 \mathrm{sec}$, annealing at $60^{\circ} \mathrm{C}$ for $1 \mathrm{~min}$ and extension at $72^{\circ} \mathrm{C}$ for $2 \mathrm{~min}$, and final extension at $72^{\circ} \mathrm{C}$ for $4 \mathrm{~min}$. The $2^{-\Delta \Delta \mathrm{Ct}}$ method was used to calculate the relative expression levels of miRNA-210 and U6.

Western blotting. Prior to protein extraction, tissue samples were homogenized using a homogenizer (PRO 200 homogenizer; Pro Scientific, Inc.). Total protein was extracted from the tissue sections and CSF using radioimmunoprecipitation assay buffer (Beyotime Institute of Biotechnology, Shanghai, China) and the protein concentration was determined using the BCA protein assay kit. Protein samples $(30 \mu \mathrm{g})$ were separated by $10 \%$ SDS-PAGE, after which they were transferred onto a polyvinylidene fluoride membrane (EMD Millipore, Billerica, MA, USA). After blocking with 5\% non-fat milk for $1 \mathrm{~h}$ at room temperature, the membrane was incubated with primary rabbit anti-human VEGF polyclonal antibody (1:1,000 dilution) and rabbit anti-human $\beta$-actin polyclonal antibody (1:5,000 dilution) at $4^{\circ} \mathrm{C}$ overnight. After washing with $150 \mathrm{mmol} / 1$ Tris-buffered saline containing 5\% Tween (Amresco, LLC, Solon, $\mathrm{OH}$, USA), the membrane was incubated with sheep anti-rabbit HRP-conjugated $\operatorname{IgG}(1: 3,000)$ at room temperature for $1 \mathrm{~h}$. Subsequently, the membrane was developed via incubation with Enhanced Chemiluminescence Reagent Plus (Abcam). The western blot images were analyzed using Image Lab 3.0 software. $\beta$-actin was used as an internal control. The relative value of VEGF was defined as the grey value ratio of VEGF: $\beta$-actin. 
A

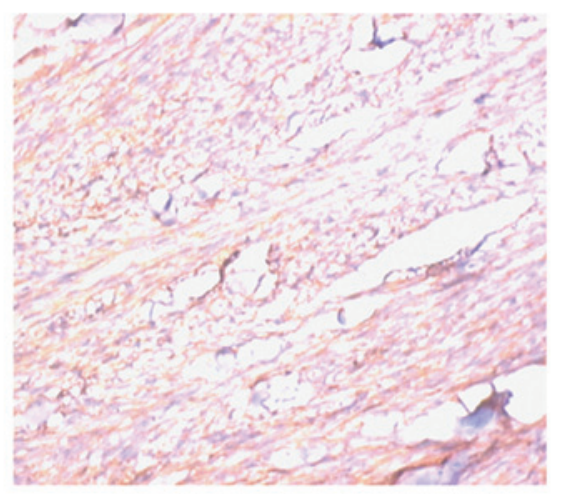

Tumor adjacent tissue of $\mathrm{MB}$

C

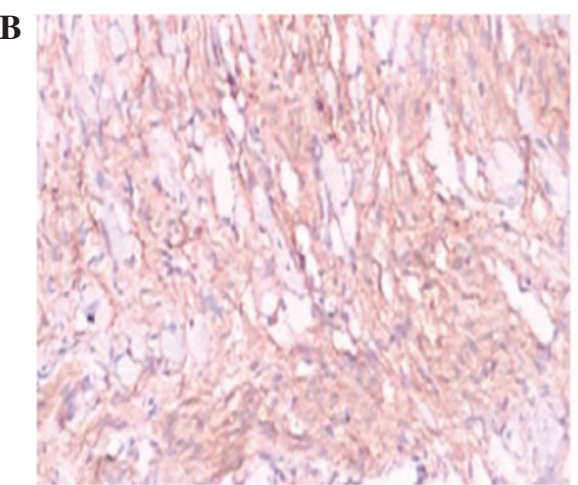

Tumor tissue of $\mathrm{MB}$

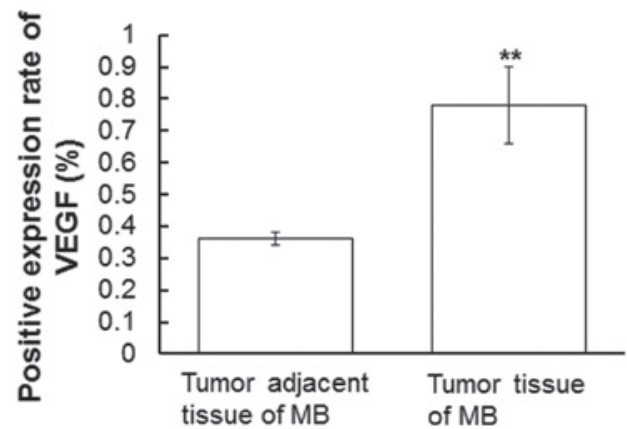

Figure 1. Immunohistochemical analysis of vascular endothelial cell growth factor (VEGF) protein expression in tumor tissues and tumor adjacent tissues from patients with medulloblastoma (MB). VEGF expression was detected using immunohistochemistry methods. Cells with brown or tan granules in the cytoplasm or on the membrane were defined as positive. Representative immunohistochemical staining results demonstrating VEGF expression in (A) tumor adjacent tissues and (B) tumor tissues of patients with MB (magnification, 400x). (C) Quantitative immunohistochemical staining results showing VEGF expression. Positive cells were counted and the positive expression rate was defined as the ratio of the number of positive cells to the total number of cells. The positive expression rate of VEGF was significantly higher in the MB tumor tissues, as compared with in the tumor adjacent tissues. Data are presented as the mean \pm standard deviation. ${ }^{* *} \mathrm{P}<0.01$ vs. the tumor adjacent tissues.

A

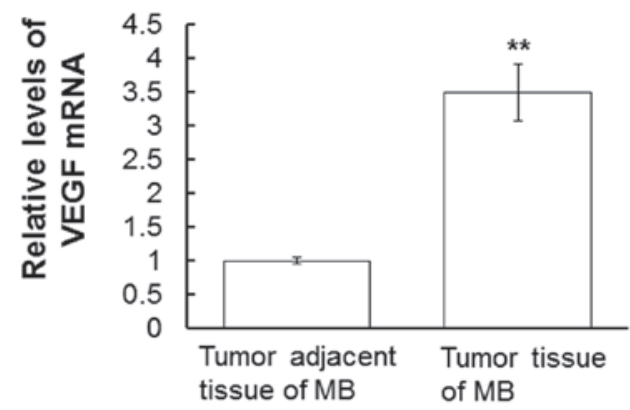

B

C
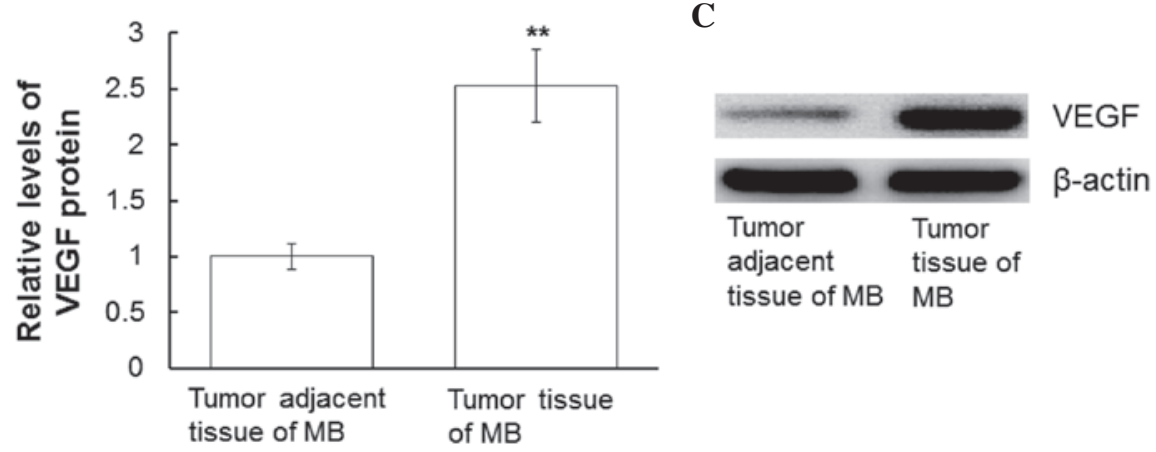

Figure 2. Analysis of vascular endothelial cell growth factor (VEGF) mRNA and protein expression levels in tumor tissues and tumor adjacent tissues from patients with medulloblastoma (MB). (A) VEGF mRNA expression levels were measured using reverse transcription-quantitative polymerase chain reaction. VEGF mRNA expression levels were significantly upregulated in MB tumor tissues, as compared with in the tumor adjacent tissues. (B and C) VEGF protein expression levels were measured using western blotting, in which $\beta$-actin was used as an internal control. VEGF protein expression levels were upregulated in the MB tumor tissues, as compared with in the tumor adjacent tissues. Data are presented as the mean \pm standard deviation. ${ }^{* *} \mathrm{P}<0.01$ vs. the tumor adjacent tissues. 
A

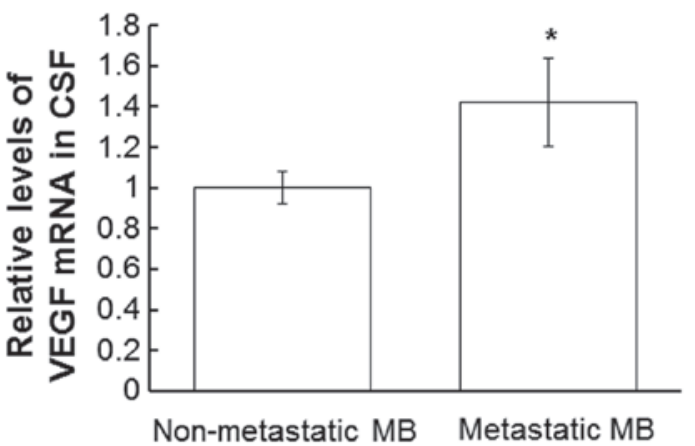

B

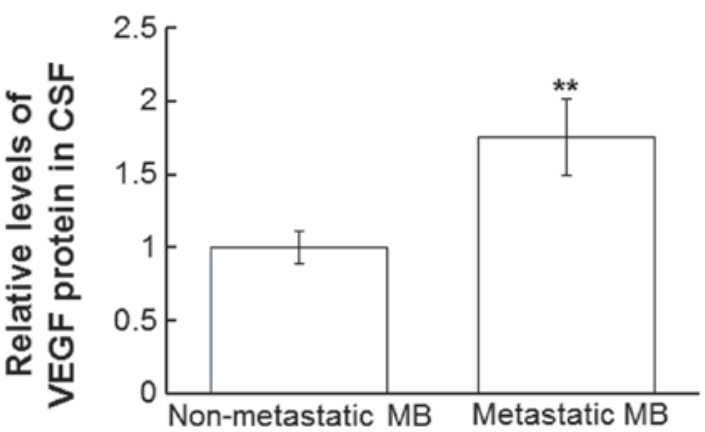

C

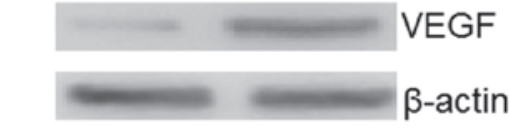

Non-metastatic MB Metastatic MB

Figure 3. Analysis of vascular endothelial cell growth factor (VEGF) mRNA and protein expression levels in the cerebral spinal fluid (CSF) of patients with primary medulloblastoma (MB; non-metastasis) and metastatic MB (MB-metastasis). (A) VEGF mRNA expression levels were measured using reverse transcription-quantitative polymerase chain reaction. VEGF mRNA expression levels were significantly upregulated in the CSF of patients with metastatic MB, as compared with non-metastatic MB. (B and C) VEGF protein expression levels were measured using western blotting, in which $\beta$-actin was used as an internal control. VEGF protein expression levels were significantly upregulated in the CSF of patients with metastatic MB, as compared with non-metastatic MB. Data are presented as the mean \pm standard deviation. ${ }^{*} \mathrm{P}<0.05$ vs. non-metastatic $\mathrm{MB} ;{ }^{* *} \mathrm{P}<0.01$. vs. non-metastatic MB.

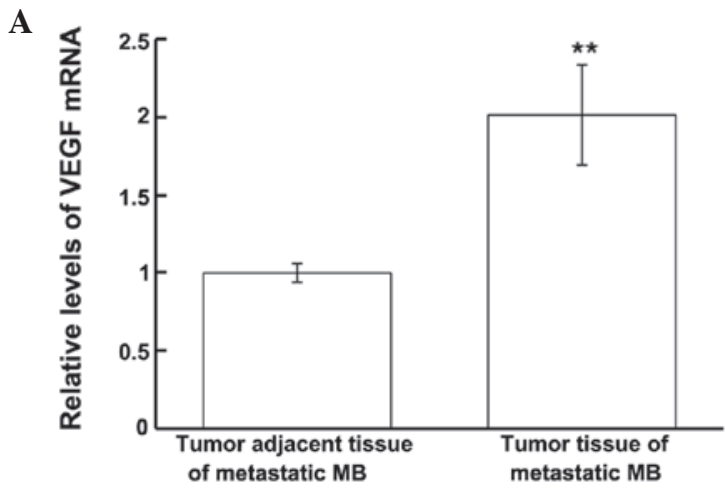

B

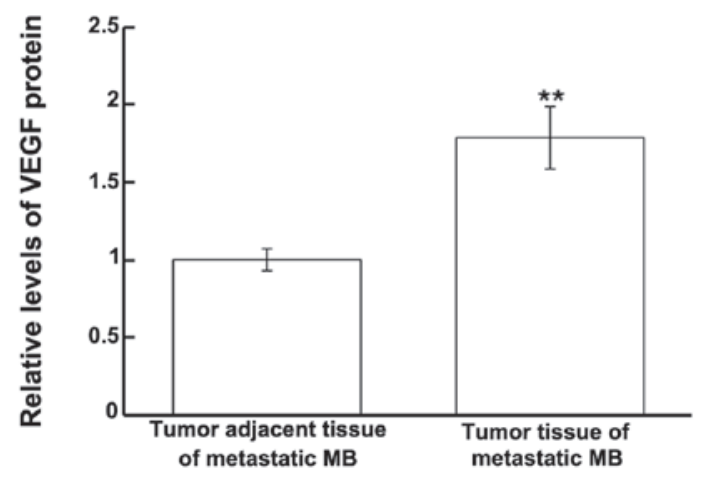

C

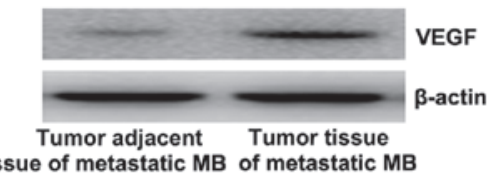

tissue of metastatic MB of metastatic MB

Figure 4. Analysis of vascular endothelial cell growth factor (VEGF) mRNA and protein expression levels in tumor and tumor adjacent tissues of patients with metastatic medulloblastoma (MB). (A) VEGF mRNA expression levels were measured using reverse transcription-quantitative polymerase chain reaction. VEGF mRNA expression levels were significantly upregulated in the tumor tissue from patients with metastatic MB, as compared with in the tumor adjacent tissues. (B and C) VEGF protein expression levels were measured using western blotting, in which $\beta$-actin was used as an internal control. VEGF protein expression levels were significantly upregulated in the tumor tissue from patients with metastatic MB, as compared with in the tumor adjacent tissues. Data are presented as the mean \pm standard deviation. ${ }^{* *} \mathrm{P}<0.01$ vs. the tumor adjacent tissues. 

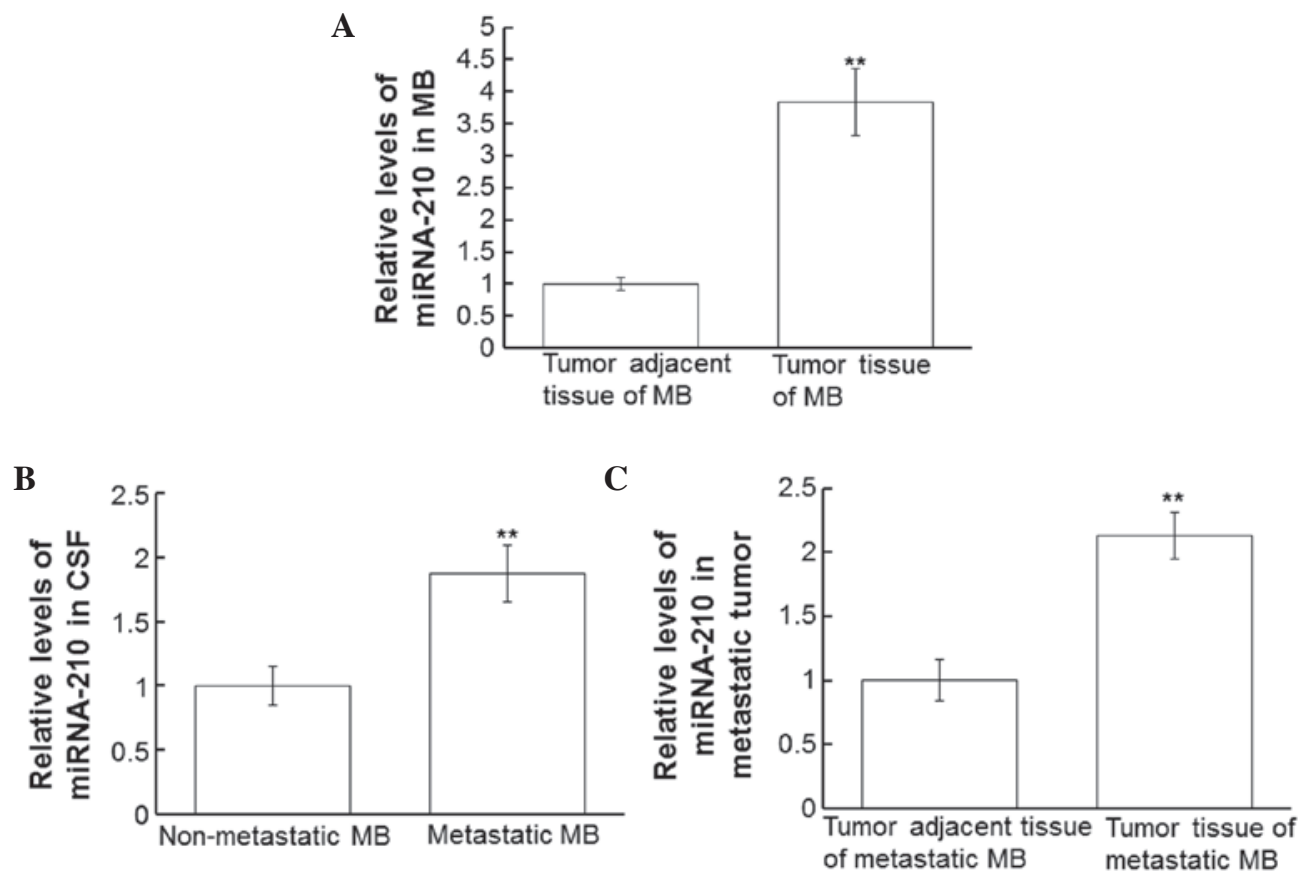

Figure 5. Analysis of micro (mi)RNA-210 expression levels. miRNA-210 expression was detected using reverse transcription-quantitative polymerase chain reaction. (A) Relative miRNA-210 expression levels in tumor and tumor adjacent tissues of patients with medulloblastoma (MB). miRNA-210 expression levels were significantly upregulated in MB tumor tissues, as compared with in tumor adjacent tissues. (B) Relative miRNA-210 expression levels in the cerebrospinal fluid (CSF) of patients with primary MB (non-metastasis) and patients with metastatic MB (MB-metastasis). miRNA-210 expression levels in the CSF of patients with metastatic MB were significantly upregulated, as compared with in the CSF of patients with non-metastatic MB. (C) Relative miRNA-210 expression levels in tumor and tumor adjacent tissues of patients with metastatic MB. miRNA-210 expression levels were significantly upregulated in the tumor tissues of patients with metastatic MB, as compared with in the tumor adjacent tissues. Data are presented as the mean \pm standard deviation. ${ }^{* *} \mathrm{P}<0.01$ vs. non-metastatic MB/tumor adjacent tissues.

Statistical analysis. SPSS 18.0 software (SPSS Inc., Chicago, IL, USA) was used for statistical analysis. Data are presented as the mean \pm standard deviation. One-way analysis of variance was performed in order to compare the differences between the various groups. $\mathrm{P}<0.05$ was considered to indicate a statistically significant difference.

\section{Results}

$V E G F$ is highly expressed in tumor tissues of $M B$. In order to determine the expression levels of VEGF in MB tumor tissues and tumor adjacent tissues, immunohistochemical analyses were performed. Representative immunohistochemical staining results are presented in Fig. 1A and B. Cells with brown or tan granules in the cytoplasm or on the membrane were defined as positive. Positive cells were counted and the positive expression rate was defined as the ratio of the number of positive cells to the total number of cells. The positive expression rate of VEGF was significantly higher in the MB tumor tissues, as compared with in the tumor adjacent tissues $(\mathrm{P}<0.01$; Fig $1 \mathrm{C})$. These results indicate that VEGF expression levels in MB tumor tissues are upregulated, as compared with tissues adjacent to the tumor.

Protein and $m R N A$ expression levels of VEGF are upregulated in the tumor tissues of patients with $M B$. In order to analyze the mRNA and protein expression levels of VEGF, RT-qPCR and western blotting of tumor and tumor adjacent tissues from patients with MB were conducted. As compared with in the tumor adjacent tissues, VEGF mRNA expression levels in the tumor tissues from patients with $\mathrm{MB}$ were significantly upregulated $(\mathrm{P}<0.01$; Fig. 2A). Concordantly, VEGF protein expression levels were significantly increased in the MB tumor tissues, as compared with in the tumor adjacent tissues $(\mathrm{P}<0.01$; Fig. 2B and C). These results suggest that VEGF mRNA and protein expression levels are upregulated in the tumor tissues of patients with MB.

Protein and mRNA expression levels of VEGF are upregulated in the CSF of patients with metastatic MB. In order to compare the expression levels of VEGF mRNA and protein in the CSF of patients with and without secondary MB tumors, RT-qPCR and western blotting were performed using CSF collected from all patients. The VEGF mRNA expression levels in the CSF from patients with metastatic MB were significantly upregulated, as compared with in the CSF from patients without secondary tumors $(\mathrm{P}<0.05$; Fig. $3 \mathrm{~A})$. In addition, VEGF protein expression levels in the CSF from patients with metastatic MB were significantly upregulated, as compared with in the CSF from patients without metastases $(\mathrm{P}<0.01$; Fig. 3B and $\mathrm{C})$. These results suggest that VEGF is upregulated in the CSF of patients with metastatic MB.

Protein and mRNA expression levels of VEGF are upregulated in tumor tissues from patients with metastatic MB. In order to investigate the mRNA and protein expression levels of VEGF in tumor tissues from patients with metastatic MB, tumor and tumor adjacent tissues from only the patients with 
secondary tumors were analyzed. VEGF mRNA and protein expression levels were measured using RT-qPCR and western blotting, respectively. The VEGF mRNA expression levels in the tumor tissues from patients with metastatic MB were significantly upregulated, as compared with in the tumor adjacent tissues $(\mathrm{P}<0.01$; Fig. 4A). Similarly, western blotting results demonstrated that VEGF protein expression levels were significantly upregulated in the tumor tissues from patients with metastatic $\mathrm{MB}$, as compared with in the tumor adjacent tissues $(\mathrm{P}<0.01$; Fig. 4B). These results indicate that VEGF mRNA and protein expression levels are upregulated in the tumors of patients with metastatic MB.

Expression levels of miRNA-210 are increased in patients with $M B$. In order to analyze the expression levels of miRNA-210 in patients with MB, RT-qPCR of MB tumor tissue, tumor adjacent tissue, CSF and metastatic tumor tissue samples was conducted. miRNA-210 expression levels in the tumor tissues of patients with MB were significantly upregulated, as compared with in the tumor adjacent tissues $(\mathrm{P}<0.01$; Fig. 5A). Similarly, miRNA-210 expression levels in the CSF from patients with metastatic $\mathrm{MB}$ were significantly increased, as compared with in the CSF from patients with primary $\mathrm{MB}(\mathrm{P}<0.01$; Fig. $5 \mathrm{~B})$. In addition, miRNA-210 expression levels in the tumor tissues of patients with metastatic MB were significantly upregulated, as compared with in the tumor adjacent tissues $(\mathrm{P}<0.01$; Fig. $5 \mathrm{C})$. These results indicate that miRNA-210 levels are upregulated in patients with $\mathrm{MB}$.

\section{Discussion}

Hypoxia is able to induce expression of VEGF and hypoxia inducible factor-1 $\alpha$, which in turn may activate downstream factors involved in tumor invasion and metastasis (16); therefore, the expression levels of VEGF may be used as an indicator for assessing tumor invasion and metastasis. In the present study, VEGF mRNA and protein expression levels were significantly upregulated in the tumor tissues of patients with MB and metastatic MB; thus indicating the occurrence of hypoxia in MB. Furthermore, upregulated VEGF may have a role in promoting tumor angiogenesis.

The detection of VEGF expression in brain tissue can, to some extent, reveal the pathological process of brain tumor invasion (17). In the present study, immunohistochemical staining demonstrated that the positive expression rate of VEGF was elevated in MB tumor tissues, as compared with in non-tumor tissues adjacent to MB. This indicates that the expression levels of VEGF may be associated with the degree of MB infiltration. The metastasis of MB primary tumors occurs predominantly via the CSF (18); therefore, the levels of VEGF in the CSF of patients with $\mathrm{MB}$ and metastatic MB were also analyzed in the present study. The results demonstrated that VEGF levels were significantly upregulated in the CSF of patients with metastatic MB, as compared with in patients with primary MB. The reason for this may be that VEGF expression increases the transcriptional activation of numerous downstream molecules, and in doing so initiates the invasion and metastasis of MB (19), ultimately leading to the metastasis of MB via the CSF.

Previous studies have detected miRNA-210-mediated regulation of VEGF expression and angiogenesis in various processes, including tumor formation and development (20), angiogenesis and nerve repair in the brain (21), capillary formation (22), and ligament repair (23). In addition, Szabó et al (24) demonstrated that VEGF mRNA is a target of miRNA-210; therefore, miRNA-210 has the potential to be used as a specific biomarker in the early diagnosis and treatment of diseases associated with VEGF (25). In the present study, consistent with the alterations in the expression levels of VEGF, miRNA-210 expression levels were elevated in the tumor tissues of patients with $\mathrm{MB}$ and metastatic $\mathrm{MB}$, and in the CSF of patients with metastatic MB. This suggests that miRNA-210 may regulate the expression of VEGF in patients with MB.

In conclusion, VEGF and miRNA-210 expression levels were upregulated in the tumor tissues of patients with MB, and most significantly in patients with metastatic MB; thus suggesting that miRNA-210 is able to regulate the metastasis of $\mathrm{MB}$ via regulation of VEGF expression.

\section{Acknowledgements}

The authors of the present study would like to thank Dr Quanxiang Wang (Department of Neurosurgery, People's Hospital of Laiwu) for his valuable help in the study design, analysis, and writing of the present study.

\section{References}

1. Sardiñas N, Marcos R, Pestaña EM, Vargas J, Chi-Ramírez D, Rojas E, Esteban EM and Zarrabeitía L: Tumors of the posterior fossa in children. Rev Neurol 28: 1153-1158, 1999 (In Spanish).

2. Packer RJ, Cogen P, Vezina G and Rorke LB: Medulloblastoma: Clinical and biologic aspects. Neuro Oncol 1: 232-250, 1999.

3. Yan XY: Angiogenesis: A promising strategy for tumor therapy. Sheng Wu Hua Xue Yu Sheng Wu Wu Li Xue Bao 26: 180-193, 2010 (In Chinese).

4. Lohela M, Bry M, Tammela T and Alitalo K: VEGFs and receptors involved in angiogenesis versus lymphangiogenesis. Curr Opin Cell Biol 21: 154-165, 2009.

5. Roberts E, Cossigny DA and Quan GM: The role of vascular endothelial growth factor in metastatic prostate cancer to the skeleton. Prostate Cancer 2013: 418340, 2013.

6. Mao X, Wang T, Liu Y, Irwin MG, Ou JS, Liao XL, Gao X, $\mathrm{Xu}$ Y, Ng KF, Vanhoutte PM and Xia Z: N-acetylcysteine and allopurinol confer synergy in attenuating myocardial ischemia injury via restoring HIF-1 $\alpha / \mathrm{HO}-1$ signaling in diabetic rats. PLoS One 8: e68949, 2013.

7. Vimala N, Mittal S, Kumar S, Dadhwal V and Sharma Y: A randomized comparison of sublingual and vaginal misoprostol for cervical priming before suction termination of first-trimester pregnancy. Contraception 70: 117-120, 2004.

8. Mende A, Takano H, Kodama Y, Nakamura T, Umetani K, Fujioka D, Saito Y, Kobayashi T, Kawabata K, Obata JE, et al: Relation between transcardiac gradient of VEGF and coronary flow response in humans. Int J Cardiol 119: 156-162, 2007.

9. Stumpf C, Jukic J, Yilmaz A, Raaz D, Schmieder RE, Daniel WG and Garlichs CD: Elevated VEGF-plasma levels in young patients with mild essential hypertension. Eur J Clin Invest 39: 31-36, 2009.

10. Xiong N, Zhang Z, Huang J, Chen C, Zhang Z, Jia M, Xiong J, Liu X, Wang F, Cao X, et al: VEGF-expressing human umbilical cord mesenchymal stem cells, an improved therapy strategy for Parkinson's disease. Gene Ther 18: 394-402, 2011.

11. Kim NH, Jung HH, Cha DR and Choi DS: Expression of vascular endothelial growth factor in response to high glucose in rat mesangial cells. J Endocrinol 165: 617-624, 2000.

12. Li H, Wang Y, Liu F, Lou YL, Deng J and Cui SP: Dynamic changes of the expression of hypoxia inducible factor- $1 \alpha$ and the target genes miR-210, vascular endothelial growth factor in the kidney after ischemic-reperfusion injury. Zhong Hua Shi Yan Wai Ke Za Zhi 28: 2074-2076, 2011 (In Chinese). 
13. Cha HS, Bae EK, Koh JH, Chai JY, Jeon CH, Ahn KS, Kim J and Koh EM: Tumor necrosis factor-alpha induces vascular endothelial growth factor- $\mathrm{C}$ expression in rheumatoid synoviocytes. J Rheumatol 34: 16-19, 2007.

14. Agrawal R, Pandey P, Jha P, Dwivedi V, Sarkar C and Kulshreshtha R: Hypoxic signature of microRNAs in glioblastoma: Insights from small RNA deep sequencing. BMC Genomics 15: 686, 2014.

15. Liu SQ, Yu HC, Gong YZ and Lai NS: Quantitiative measurement of HLA-B27 mRNA in patients with ankylosing spondylitis - correlation with clinical activity. J Rheumatol 33: 1128-1132, 2006

16. Brahimi-Horn MC, Chiche J and Pouysségur J: Hypoxia and cancer. J Mol Med (Berl) 85: 1301-1307, 2007.

17. Cohen AL and Colman H: Glioma biology and molecular markers. Cancer Treat Res 163: 15-30, 2015.

18. Barai S, Bandopadhayaya GP, Julka PK, Dhanapathi H, Haloi AK and Seith A: Cerebellar medulloblastoma presenting with skeletal metastasis. J Postgrad Med 50: 110-112, 2004.

19. Slongo ML, Molena B, Brunati AM, Frasson M, Gardiman M Carli M, Perilongo G, Rosolen A and Onisto M: Functional VEGF and VEGF receptors are expressed in human medulloblastomas. Neuro Oncol 9: 384-392, 2007.
20. Quero L, Dubois L, Lieuwes NG, Hennequin C and Lambin P: miR-210 as a marker of chronic hypoxia, but not a therapeutic target in prostate cancer. Radiother Oncol 101: 203-208, 2011.

21. Zeng L, He X, Wang Y, Tang Y, Zheng C, Cai H, Liu J, Wang Y, Fu Y and Yang GY: MicroRNA-210 overexpression induces angiogenesis and neurogenesis in the normal adult mouse brain. Gene Ther 21: 37-43, 2014.

22. Lou Y, Gao F, Xie A, Guo F, Deng Z and Wang Y: MicroRNA-210 modified human umbilical vein endothelial cells induce capillary formation. Zhongguo Xiu Fu Chong Jian Wai Ke Za Zhi 26: 587-591, 2012 (In Chinese).

23. Shoji T, Nakasa T, Yamasaki K, Kodama A, Miyaki S, Niimoto T, Okuhara A, Kamei N, Adachi N and Ochi M: The effect of intra-articular injection of microRNA-210 on ligament healing in a rat model. Am J Sports Med 40: 2470-2478, 2012.

24. Szabó DR, Luconi M, Szabó PM, Tóth M, Szücs N, Horányi J, Nagy Z, Mannelli M, Patócs A, Rácz K and Igaz P: Analysis of circulating microRNAs in adrenocortical tumors. Lab Invest 94: 331-339, 2014

25. Alaiti MA, Ishikawa M, Masuda H, Simon DI, Jain MK, Asahara T and Costa MA: Up-regulation of miR-210 by vascular endothelial growth factor in ex vivo expanded $\mathrm{CD} 34^{+}$cells enhances cell-mediated angiogenesis. J Cell Mol Med 16: 2413-2421, 2012. 